\begin{tabular}{|c|l|}
\hline Title & Photoexpansion in A s2S3 glass \\
\hline Author(s) & Tanaka, Keiji \\
\hline Citation & $\begin{array}{l}\text { PHY SICAL REVIEW B, 57(9), 5163-5167 } \\
\text { https://doi.org/L0.1103/PhysRevB.57.5163 }\end{array}$ \\
\hline Issue Date & 1998-03 \\
\hline Doc URL & http://hdl.handle.net/2115/5789 \\
\hline Rights & Copyright $\odot$ 1998 A merican Physical Society \\
\hline Type & article \\
\hline File Information & PRB57-9.pdf \\
\hline
\end{tabular}

Instructions for use 


\title{
Photoexpansion in $\mathrm{As}_{2} \mathrm{~S}_{3}$ glass
}

\author{
Keiji Tanaka \\ Department of Applied Physics, Faculty of Engineering, Hokkaido University, Sapporo 060, Japan
}

(Received 30 June 1997)

\begin{abstract}
Mechanisms of photoexpansion in chalcogenide glasses have been studied in three respects. A detailed x-ray investigation of $\mathrm{As}_{2} \mathrm{~S}_{3}$ shows that the photoexpansion can be connected with asymmetric broadening of the first sharp diffraction peak. Comparison between radiation-induced volume changes and density ratios of glassyto-crystalline forms in $\mathrm{As}_{2} \mathrm{~S}_{3}$ and $\mathrm{SiO}_{2}$ implies that $\mathrm{As}_{2} \mathrm{~S}_{3}$ can expand since the glass is as dense as its crystal. When $\mathrm{As}_{2} \mathrm{~S}_{3}$ is exposed to illumination, the photoexpansion appears earlier and later than the photodarkening for bandgap and subbandgap illumination, respectively. These observations are discussed from a microscopic point of view. [S0163-1829(98)09209-1]
\end{abstract}

\section{INTRODUCTION}

Photoinduced phenomena in chalcogenide glasses have attracted considerable interest because of their scientific and technological importance., ${ }^{1,2}$ In scientific respect, the phenomena are assumed to be caused through electron-lattice interaction in metastable disordered atomic structures, the subject not yet being understood. In addition, the phenomena are promising for optical applications. For instance, the photoinduced phase change discovered by Ovshinsky's group $^{3}$ has been developed to erasable optical mass memories. ${ }^{4}$

Among a variety of phenomena, reversible photodarkening is one of the most extensively studied. ${ }^{1,2,5}$ When a chalcogenide glass, which may be elemental or compound, is exposed to light, it shows a redshift of the optical-absorption edge, which can be recovered with annealing at the glasstransition temperature. Bandgap light is effective in producing the redshift, ${ }^{1,5}$ while subbandgap light can also induce some changes. ${ }^{1,2,6}$ The photodarkening phenomenon is demonstrated to arise from photoinduced enhancement of randomness in amorphous structures, ${ }^{7}$ while the entity of atomic change is still speculative. ${ }^{1,2}$

Chalcogenide glasses also exhibit volume changes upon light illumination. ${ }^{1,2}$ Hamanaka et al. have discovered that when a chalcogenide glass is photodarkened by bandgap illumination, the material exhibits a macroscopic expansion, whereupon it is recovered with annealing. ${ }^{8-10}$ The fractional expansion $\Delta V / V$ is, for instance, about $0.5 \%$ in $\mathrm{As}_{2} \mathrm{~S}_{3}$ illuminated at room temperature. ${ }^{8,9,11,12}$ In addition, Hisakuni and Tanaka have recently demonstrated that, upon illumination of subbandgap light, chalcogenide glasses exhibit more dramatic expansion, which can be referred to as "giant photoexpansion.", 13 For instance, $\mathrm{As}_{2} \mathrm{~S}_{3}$ exposed at room temperature to light emitted from a He-Ne laser exhibits volume expansions of $\sim 5 \%$, which can be utilized as microlenses. ${ }^{14}$ It may be valuable to mention here that, in $\mathrm{SiO}_{2}$ glass, irradiation gives rise to an opposite effect, "radiation compaction,", 15-17 i.e., densification induced by irradiation, which can also be thermally recovered.

Then, we may pose some questions. What kind of atomic changes is responsible for the photoexpansion? Why do $\mathrm{As}_{2} \mathrm{~S}_{3}$ and $\mathrm{SiO}_{2}$ show the opposite volume changes upon irradiation? Do the photodarkening and the photoexpansion, both being induced by bandgap and subbandgap illumination, arise from a single mechanism? The present work will deal with these problems.

\section{EXPERIMENTS}

The samples investigated were $\mathrm{As}_{2} \mathrm{~S}_{3}$ glass in bulk forms and films. Bulk samples were prepared through the conventional melt-quenching and polishing procedures. Films were obtained by evaporating the bulk ingots onto substrates of oxide glasses (for optical and expansion measurements) and $\mathrm{Al}$ foils and $\mathrm{Si}$ wafers (for X-ray measurements). Thicknesses were 1-50 $\mu \mathrm{m}$. These samples were annealed at the glasstransition temperature before measurements. Then, the samples were exposed to bandgap light provided from a 250 $\mathrm{W}$ ultrahigh pressure $\mathrm{Hg}$ lamp and an Ar laser, and to subbandgap light from a He-Ne laser.

Photoinduced effects were evaluated in three properties. Optical transmittance was measured using a conventional double-beam spectrometer (JASCO, V-570S). Photoexpansion was measured using a surface profile-meter (TENCOR, $\alpha$-step) and an atomic-force microscope (Burleigh, Aris 3300). X-ray-diffraction patterns were obtained using a diffractometer system consisting of a rotating $\mathrm{Cu}$ anode (Rigaku, RU-300) and a proportional counter fitted with a graphite monochromator. In some measurements, the detector was replaced to a position-sensitive proportional counter.

\section{RESULTS AND DISCUSSION}

\section{A. Origin of the photoexpansion}

In order to know an atomic mechanism of the photoexpansion, detailed x-ray-diffraction patterns before and after light illumination have been measured. Figure 1 shows a typical result. We see in the upper pattern that the first sharp diffraction peak (FSDP) is located at the wave number $Q$ $\simeq 1.2 \AA^{-1}$, consistent with previous studies. ${ }^{5,7,18}$ The lower pattern shows the intensity difference between the annealed and the illuminated state, where positive differences indicate decreases in the x-ray intensity with illumination.

Two kinds of photoinduced FSDP changes should be marked. First, angular shifts of the peak position, which are 


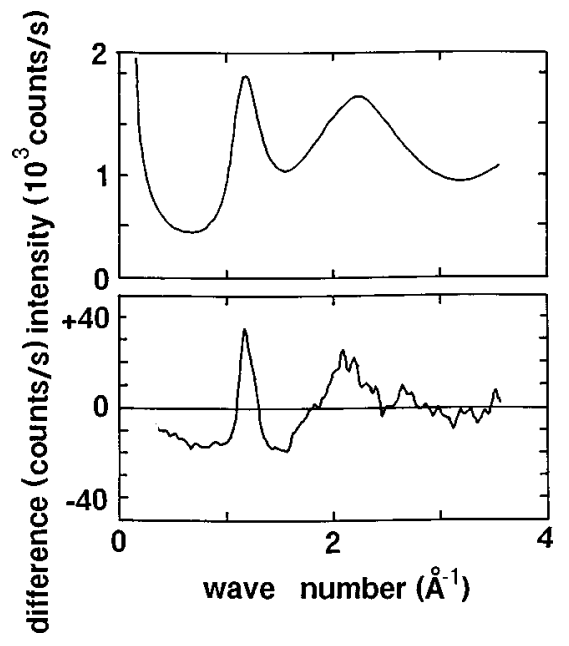

FIG. 1. An x-ray diffraction pattern of an annealed $\mathrm{As}_{2} \mathrm{~S}_{3}$ bulk sample (upper) and the intensity decrease (lower) induced with illumination from an $\mathrm{Hg}$ lamp for $1 \mathrm{~h}$. Positive difference means intensity reduction with illumination.

prominent for samples illuminated at low temperatures, ${ }^{7,19}$ are not detected, or very small ${ }^{20}$ the result being consistent with Ref. 21. Second, the peak becomes weaker and broader with illumination, and at peak tails intensity enhancements occur. In more detail, we see that the photoinduced intensity enhancement (negative difference) at the peak tails is not symmetric. That is, the intensity enhancement at $Q$ $\simeq 0.8 \AA^{-1}$ appears to be broader than that at $\sim 1.5 \AA^{-1}$.

Since the asymmetric photoinduced FSDP change is very small, reproducibility of the result has been examined carefully. For instance, $\mathrm{As}_{2} \mathrm{~S}_{3}$ samples were illuminated in situ on goniometers. And, the asymmetric change was investigated for three kinds of samples; films deposited on Si substrates and polished bulk samples in a reflection (Bragg) arrangement, and films on Al-foil substrates in a transmission (Laue) arrangement. All the samples exhibited similar tail changes. However, x-ray intensity at $Q \leqslant 0.3 \AA^{-1}$ was not reproducible, probably due to mechanical noise.

We here try to extract some insight from the asymmetric change under an assumption that the FSDP governs the macroscopic density. Actually, it is demonstrated through pressure studies that the changes in the FSDP position and the macroscopic density are quantitatively correlated. ${ }^{22}$ Accordingly, a similar situation can possibly be envisaged for the photoinduced phenomenon.

To perform some calculation, an assumption for origins of the FSDP is needed. The subject is still controversial, ${ }^{23-25}$ while the so-called distorted-layer model originally proposed by Vaipolin and Porai-Koshit ${ }^{18}$ can be employed as a basis for the present calculation. In the model, the FSDP is interpreted as a Bragg peak reflected from stacked distorted layers, consisting of As-S bonds, which are held together with weak forces of van der Waals character, ${ }^{18,25}$ the overall structure resembling crumpled papers. Hence, in this model, the FSDP position of $1.2 \AA^{-1}$ is interpreted to represent the interlayer distance $D$ of $\sim 5 \AA$, and the FSDP width is understood on the basis of the Scherrer equation ${ }^{26}$ to be a manifestation of the correlated dimension of stacked regions, $\sim 20 \AA .{ }^{27}$
Then, we can analyze in the following way: The macroscopic density change $\Delta V / V$ is proportional to $\Delta D / D$ $\simeq-\int Q \Delta I(Q) d Q / \int Q I(Q) d Q$, where $I(Q)$ is the FSDP intensity in the annealed state, and $\Delta I(Q)$ is the photoinduced change. ${ }^{28}$ The integral has been performed over the FSDP, $0.3-2.1 \AA^{-1},{ }^{29}$ which gives $\Delta D / D \simeq 1 \pm 0.5 \%$, where the error is mainly due to sample-to-sample variation. This ratio is comparable to the experimental observations of the photoexpansion, $\Delta V / V \simeq 0.5 \%,{ }^{8,9,12}$ despite the rough estimation procedure. In detail, we must take into account $\mathrm{X}$-ray changes in the whole wave number, specifically in smaller wavenumber regions, the proportionality factor between $\Delta V / V$ and $\Delta D / D,{ }^{22}$ and so forth.

Although the above analysis is preliminary, the quantitative agreement between the $\mathrm{x}$-ray and the macroscopic change implies that the increase in structural randomness appearing as the asymmetric FSDP change governs the volume expansion. Since the FSDP central position does not change, the typical interlayer distance of $\sim 5 \AA^{-1}$ seems to be intact. However, the x-ray intensity enhancement at $Q \simeq 0.8 \AA^{-1}$ implies that at a few places the interlayer distance becomes as wide as $\sim 10 \AA$. We may envisage the generation of inhomogeneous structures containing some interlayer cracks.

Similar structural changes may be induced by subbandgap illumination, while reproducible FSDP changes could not been detected. This was mostly due to more stringent experimental situations. For subbandgap illumination provided from He-Ne lasers the light beam should be focused onto spots of $\sim 0.1 \mathrm{~mm}^{2},{ }^{13}$ and accordingly small $\mathrm{x}$-ray changes could not be detected accurately, despite an efficient counting system including a position-sensitive detector.

It seems fair to discuss another possibility which may give rise to the volume expansion. Regarding structural changes induced by illumination, it is demonstrated through vibrational studies that heteropolar bonds are converted to homopolar bonds, i.e., As-S to As-As and S-S. ${ }^{1,30,31}$ The conversion may cause the volume expansion, since the bond length of As-As [2.57 $\AA$ (Ref. 32)] is longer than that of As-S [2.24 $\AA$ (Ref. 32)]. However, the S-S length is reported to be shorter [2.05 $\AA$ (Ref. 32)], and accordingly it is not necessarily evident if the heteropolar-to-homopolar bond conversion can produce the volume expansion. In addition, in $\mathrm{GeS}_{2}$, which is also known to exhibit photoinduced volume expansion (see Table I), such heteropolar-to-homopolar bond conversion has not been detected through Ramanscattering studies. ${ }^{33}$ Therefore, it seems less probable that the bond conversion causes the volume expansion.

Photoinduced creation of voids is also plausible, which may be detected using small-angle x-ray scattering. However, we could not obtain reproducible features in X-ray intensity at $Q \simeq 0.1 \AA^{-1}$. It is mentioned here that void concentration in $\mathrm{As}_{2} \mathrm{~S}(\mathrm{Se})_{3}$ has been demonstrated to be small and stable ${ }^{34,35}$ while no data are available for the photoinduced change.

\section{B. Radiation effects on density in chalcogenide and oxide glasses}

Table I summarizes volume changes induced by some irradiation in chalcogenide and oxide glasses at room temperature. These volume changes are assumed or demonstrated to 
TABLE I. Volume changes in some chalcogenide glasses, oxide glasses, and crystals induced with irradiation denoted. Glass and crystal are abbreviated to $g$ - and $c$-. Also shown by $\rho_{g}$ and $\rho_{c}$ are the glass density and the density of the corresponding crystal. The ratio is given as $R=\rho_{g} / \rho_{c}$.

\begin{tabular}{llllrl}
\hline \hline Material & \multicolumn{1}{c}{ Excitation } & \multicolumn{1}{c}{$\Delta V / V(\%)$} & $\rho_{g}\left(\mathrm{~g} / \mathrm{cm}^{3}\right)$ & $\rho_{c}\left(\mathrm{~g} / \mathrm{cm}^{3}\right)$ & $R$ \\
\hline$g-\mathrm{As}_{2} \mathrm{~S}_{3}$ & Light & +0.4 (Refs. 8, 9) & 3.2 (Ref. 45) & 3.43 (Ref. 46) & 0.93 \\
& Light & +0.7 (Ref. 12) & & & \\
$g-\mathrm{As}_{2} \mathrm{Se}_{3}$ & Light & +0.7 (Ref. 12) & 4.58 (Ref. 47) & 4.75 (Ref. 46) & 0.96 \\
$g-\mathrm{GeS}_{2}$ & Light & +0.5 (Ref. 10) & 2.7 (Ref. 48) & 2.94 (Ref. 46) & 0.92 \\
$g-\mathrm{SiO}_{2}$ & E-beam & -3 (Ref. 49) & 2.2 (Ref. 46) & 2.65 (Ref. 46) & 0.83 \\
& Neutron & -3 (Ref. 39) & & \\
$g-\mathrm{Ge}_{1} \mathrm{As}_{4} \mathrm{Se}_{5}$ & Light & -0.01 (Ref. 50) & & & \\
$g-\mathrm{SiO}_{2}-\mathrm{Na}_{2} \mathrm{O}$ & Light & + (Ref. 17) & & & \\
$c-\mathrm{SiO}_{2}$ & Neutron & +15 (Ref. 39) & & & \\
& E-beam & +10 (Ref. 49) & & & \\
$c-\mathrm{KBr}$ & X ray & +0.0001 (Ref. 37) & & & \\
\hline \hline
\end{tabular}

be reversible, i.e., the changes can be recovered with annealing. We see that the volume changes in simple chalcogenide glasses and $\mathrm{SiO}_{2}$ are opposite; upon irradiation the chalcogenide expands and the oxide contracts. However, in both cases, diffraction studies show that irradiation always gives rise to randomness enhancements in amorphous structures, specifically at around the FSDP's, as demonstrated for chalcogenide glasses in Refs. 7, 19, 21 and Fig. 1 and for $\mathrm{SiO}_{2}$ in Ref. 36. (It is mentioned here that in $\mathrm{SiO}_{2}$ the FSDP shifts to higher angles, in harmony with the volume contraction.)

It is known that in ionic crystals irradiation always induces volume expansion, some examples being listed in Table I. The volume expansion has been understood to be a manifestation of the creation of point defects such as those of the Schottky type. ${ }^{37}$ This result suggests that the randomness enhancement in ordered structures causes volume expansion.

Following this notion, we list in Table I the densities of crystalline and glassy chalcogenides and $\mathrm{SiO}_{2}$ and its ratio $R\left(=\rho_{g} / \rho_{c}\right)$. Here, $R$ can be regarded as a measure of the compactness of glassy structures in comparison with that of the corresponding crystalline structures, which are assumed to be the most compact.

We see that in the stoichiometric chalcogenide glasses $R$ $\geqslant 0.9$, while $R \simeq 0.8$ in $\mathrm{SiO}_{2}$. This greater $R$ value in the chalcogenide glasses implies that the structure is fairly ordered, and accordingly irradiation can increase the structural randomness, which causes volume expansion, as is the case in the ionic crystals. In contrast, in $\mathrm{SiO}_{2}$ the structure is less dense and more disordered, probably due to threedimensional random network structures. ${ }^{36,38}$ Accordingly, irradiation can give the densification with mechanisms presently speculative. ${ }^{15-17}$

We can also offer coherent explanation for a few exceptional cases listed in Table I. For $\mathrm{Ge}_{1} \mathrm{As}_{4} \mathrm{Se}_{5}$, a ternary nonstoichiometric glass, the structure is assumed to be fairly random and three-dimensional. ${ }^{38}$ Thus, it exhibits the photodensification, as $\mathrm{SiO}_{2}$ does. On the other hand, $\mathrm{SiO}_{2}-\mathrm{Na}_{2} \mathrm{O}$ can be assumed to be fairly dense, since the $\mathrm{Na}_{2} \mathrm{O}$ network modifiers fill free spaces in the three-dimensional random structure. ${ }^{17,39}$ Hence, it can expand accompanying randomness enhancement.

\section{Photoexpansion and photodarkening in $\mathrm{As}_{2} \mathrm{~S}_{3}$}

In order to investigate the relationship between the photoexpansion $\Delta V$ and the photodarkening $\Delta E$, dependence on exposure time has been measured. Figure 2 shows the results for $\Delta L$, which is equivalent to $\Delta V$ since the expansion occurring at free surfaces is measured, and $\Delta E$ upon bandgap and subbandgap illumination.
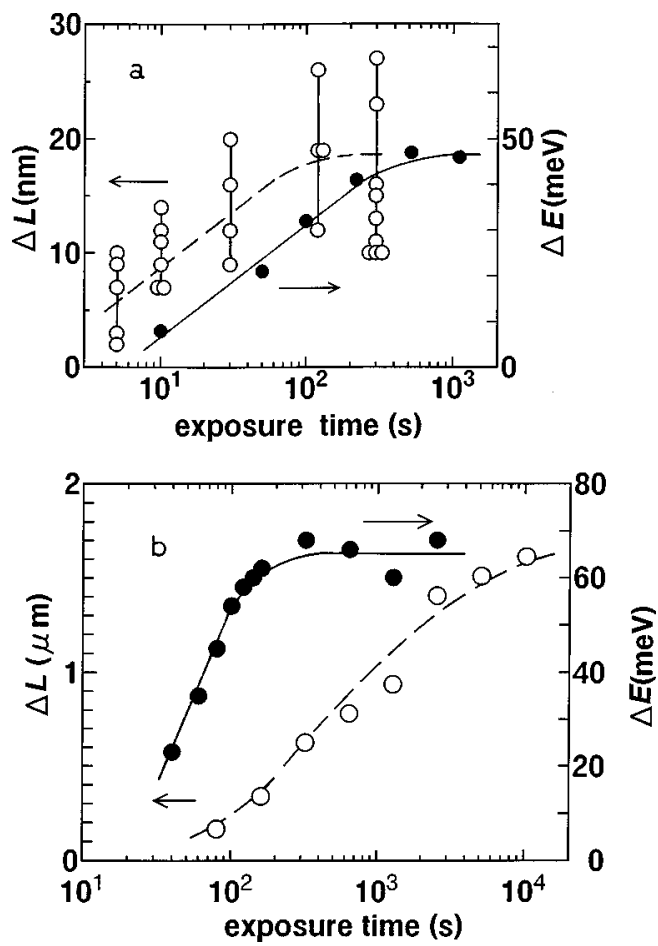

FIG. 2. The photoexpansion $\Delta L$ and the photodarkening $\Delta E$ as a function of exposure times in $\mathrm{As}_{2} \mathrm{~S}_{3}$ for bandgap (a) and subbandgap (b) illumination. $\Delta L$ is evaluated at the height of expansion, and $\Delta E$ denotes the redshift of the optical-absorption edge. The samples in (a) and (b) are an $\mathrm{As}_{2} \mathrm{~S}_{3}$ film with a thickness of $3 \mu \mathrm{m}$ and a bulk $\mathrm{As}_{2} \mathrm{~S}_{3}$ flake with a thickness of $50 \mu \mathrm{m}$. Light sources are (a) an $\mathrm{Ar}$ laser $(\hbar \omega=2.4 \mathrm{eV})$ and (b) a He-Ne laser $(\hbar \omega$ $=2.0 \mathrm{eV}$ ). 


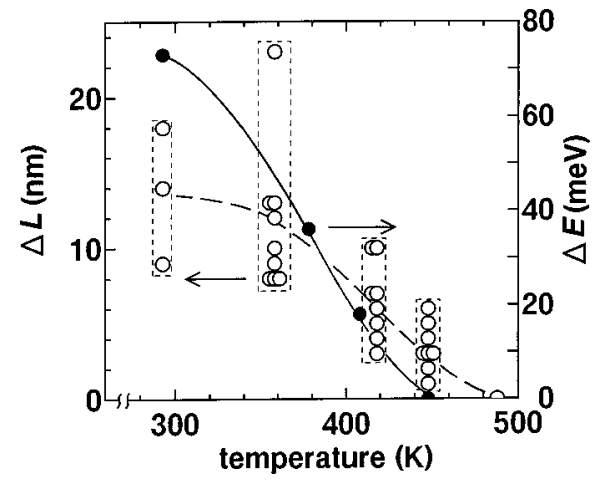

FIG. 3. Isochronal annealing characteristics of the photoexpansion $\Delta L$ and the photodarkening $\Delta E$. The sample is an $\mathrm{As}_{2} \mathrm{~S}_{3}$ film with a thickness of $3 \mu \mathrm{m}$, which has been exposed to $2.54 \mathrm{eV}$ light, and it is annealed for $1 \mathrm{~h}$ successively at the higher temperatures indicated.

We see in Fig. 2(a) that for bandgap illumination ( $\hbar \omega$ $=2.4 \mathrm{eV}$ ) $\Delta L$ increases earlier than $\Delta E$. Actually, if time constant is evaluated at the exposure time needed to induce half of the total change, the time constant for $\Delta L$ is shorter by about an order than that for $\Delta E$. Note that similar results have been obtained also for bandgap illumination provided from the Ar laser with $\hbar \omega=2.54 \mathrm{eV}$ and the Hg lamp emitting polychromatic blue light. In contrast, as shown in Fig. 2(b), for subbandgap illumination $(\hbar \omega=2.0 \mathrm{eV}), \Delta L$ increases more gradually than $\Delta E$. We might assume that the photoexpansion and the photodarkening arose from single structural changes, while the above results clearly demonstrate that the phenomena are not in one-to-one correspondence. ${ }^{42}$

The noncorrespondence between $\Delta L$ and $\Delta E$ is also seen in their annealing kinetics. Figure 3 shows the isochronal annealing characteristics for $\Delta L$ and $\Delta E$ in $\mathrm{As}_{2} \mathrm{~S}_{3}$, which has been exposed beforehand to $2.54 \mathrm{eV}$ light. Annealing is performed successively for $1 \mathrm{~h}$ at higher temperatures, and $\Delta L$ and $\Delta E$ are measured at room temperature. We see that $\Delta E$ disappears completely with annealing at $450 \mathrm{~K}$, while $\Delta L$ disappears at $490 \mathrm{~K}$. This characteristic implies that the photoexpansion is a manifestation of structural changes in a wider scale. For subbandgap illumination, $\Delta L$ is appreciably greater as shown in Fig. 2(b), and accordingly complete thermal erasure has been difficult. In fact, even with annealing at $500 \mathrm{~K}$ for $1 \mathrm{~h}$, we can still find a trace of an illuminated spot.

The feature that the photoexpansion and the photodarkening in $\mathrm{As}_{2} \mathrm{~S}_{3}$ do not correspond to each other has also been demonstrated in a pressure study. ${ }^{11}$ That is, the volume expansion of $\sim 0.5 \%$ at $1 \mathrm{~atm}$ is almost completely suppressed by application of 2 kbars hydrostatic pressure, while the compression enhances the photodarkening. At higher pressures, the photodarkening becomes smaller, ${ }^{40,41}$ and probably the photoexpansion does not appear since amorphous structures are fully compressed.

The exposure-time dependence under bandgap illumination shown in Fig. 2(a) appears anomalous, and we here speculate about that. The photodarkening is assumed to be induced by some atomic changes in a small scale, such as the bond-twisting motion of chalcogen atoms. ${ }^{1,2}$ Strains can be produced through the atomic change, which may give rise to the redshift of the optical-absorption edge. However, the strain can possibly be relaxed with interlayer relaxation, e.g., widening, which appears as the photoexpansion. A study on transient characteristics implies that the structural relaxation may occur with a time scale of $\mu \mathrm{s}{ }^{43}$ The interlayer widening can also produce some free spaces, where the atomic change responsible for the photodarkening can occur furthermore. Accordingly, the overall photodarkening may appear seemingly later than the photoexpansion.

For the result induced by subbandgap illumination shown in Fig. 2(b), photoinduced fluidity seems to add an important role. Hisakuni and Tanaka have demonstrated that the giant photoexpansion, i.e., the photoexpansion induced by subbandgap illumination, is caused by the conventional photoexpansion and the photoinduced fluidity. ${ }^{13}$ That is, since subbandgap light can penetrate into a deep interior of a sample, i.e., $\sim 100 \mu \mathrm{m}$, the photoexpansion there must be transferred to irradiated free surfaces through the photoinduced fluidity, which may be induced through interlayer slippage and bond interchanges. ${ }^{44}$ On the basis of this model, the delayed increase in $\Delta L$ under subbandgap illumination can be interpreted to be a manifestation of the longer strain-conversion process through the photoinduced fluidity.

\section{SUMMARY}

Mechanism of the photoexpansion in $\mathrm{As}_{2} \mathrm{~S}_{3}$ glass has been studied from three respects. First, detailed X-ray investigation which assumes the distorted-layer model suggests that the photoexpansion is caused by the increase in structural randomness accompanying the widening of the interlayer distance in some places. Second, the reason why $\mathrm{As}_{2} \mathrm{~S}_{3}$ exhibits the photoinduced volume expansion, in contrast to the radiation compaction in $\mathrm{SiO}_{2}$, has been considered. The expansion seems to appear in glasses possessing compact structures comparable to crystalline structures. In contrast, $\mathrm{SiO}_{2}$ seems to have a greater free volume due to three-dimensional structures, and accordingly it exhibits radiation compaction. Third, it has been demonstrated that the photoexpansion and the photodarkening do not appear simultaneously. For bandgap illumination, the photoexpansion appears earlier than the photodarkening, which may imply that the interlayer widening can produce additional sites which are responsible for successive photodarkening processes. In contrast, for subbandgap illumination, the photoexpansion appears later. Photoinduced fluidity occurring over long distances are possibly responsible for the delayed photoexpansion.

\section{ACKNOWLEDGMENTS}

The present work was supported in part with grants from the Ministry of Education and Yazaki Foundation. 
${ }^{1}$ K. Shimakawa, A. Kolobov, and S. R. Elliott, Adv. Phys. 44, 475 (1995).

${ }^{2}$ Ke. Tanaka, Solid State Mater. Sci. 1, 567 (1996).

${ }^{3}$ S. R. Ovshinsky and H. Fritzsche, IEEE Trans. Electron Devices ED-20, 91 (1973).

${ }^{4}$ T. Ohta, N. Akahira, S. Ohara, and I. Satoh, Optoelectron., Devices Technol. 10, 361 (1995).

${ }^{5}$ J. P. De Neufville, S. C. Moss, and S. R. Ovshinsky, J. NonCryst. Solids 13, 191 (1973/74).

${ }^{6}$ Ke. Tanaka, Solid State Commun. 34, 201 (1980).

${ }^{7}$ K. Tanaka, Appl. Phys. Lett. 26, 243 (1975).

${ }^{8}$ H. Hamanaka, K. Tanaka, A. Matsuda, and S. Iizima, Solid State Commun. 19, 499 (1976).

${ }^{9}$ H. Hamanaka, K. Tanaka, and S. Iizima, Solid State Commun. 23, 63 (1977).

${ }^{10}$ H. Hamanaka, K. Tanaka, and S. Iizima, Solid State Commun. 33, 355 (1980).

${ }^{11}$ Ke. Tanaka, Phys. Rev. B 30, 4549 (1984).

${ }^{12}$ M. D. Mikhailov, E. A. Karpova, Z. Cimpl, and F. Kosek, Phys. Status Solidi A 117, 467 (1990).

${ }^{13}$ H. Hisakuni and Ke. Tanaka, Appl. Phys. Lett. 65, 2925 (1994).

${ }^{14}$ H. Hisakuni and Ke. Tanaka, Opt. Lett. 20, 958 (1995).

${ }^{15}$ W. Primak and R. Kampwirth, J. Appl. Phys. 39, 5651 (1968).

${ }^{16}$ M. Rajaram and E. J. Friebele, J. Non-Cryst. Solids 108, 1 (1989).

${ }^{17}$ A. R. Silin, J. Non-Cryst. Solids 129, 40 (1991).

${ }^{18}$ A. A. Vaipolin and E. A. Porai-Koshits, Sov. Phys. Solid State 5, 497 (1963).

${ }^{19}$ H. Hamanaka, S. Minomura, and K. Tsuji, J. Non-Cryst. Solids 137\&138, 977 (1991)

${ }^{20}$ If $\mathrm{As}_{2} \mathrm{~S}_{3}$ is exposed at low temperatures and x-ray-diffraction patterns are taken at room temperature, thermal expansion may cause some influences. Thus, in the present work both are done at room temperature.

${ }^{21}$ H. Hamanaka, K. Tanaka, K. Tsuji, and S. Minomura, J. Phys. Colloq. 42, C4-399 (1981).

${ }^{22}$ Ke. Tanaka, Solid State Commun. 58, 469 (1986); in Disordered Systems and New Materials, edited by M. Borissov, N. Kirov, and A. Vavrek (World Scientific, Singapore, 1989), pp. 290309.

${ }^{23}$ S. R. Elliott, Nature (London) 354, 445 (1991).

${ }^{24}$ D. L. Price, Solid State Mater. Sci. 1, 572 (1996).

${ }^{25}$ P. H. Gaskell and D. J. Wallis, Phys. Rev. Lett. 76, 66 (1996).

${ }^{26}$ A. Guinier, X-ray Diffraction (Freeman, San Francisco, 1963), Chap. 5.

${ }^{27}$ Note that if only the size of correlated structures reduces with illumination, the peak broadening is symmetric in wave number (Ref. 26).

${ }^{28}$ Here, on the basis of continuous random network models, we may follow an analysis such as proposed in Ref. 36. However, different from $\mathrm{SiO}_{4 / 2}$ units, $\mathrm{AsS}_{3 / 2}$ units are pyramidal, and accordingly the analysis is not straightforward.

${ }^{29}$ In Fig. 1, the FSDP appears to be convoluted with other peaks, and accordingly in this calculation it is represented with a single peak with a center at $1.2 \AA^{-1}$ and a half width of $\pm 0.2 \AA^{-1}$. This simplification has little effect upon the $\Delta D / D$ value.

${ }^{30}$ M. Frumar, A. P. Firth, and A. E. Owen, Philos. Mag. B 50, 463 (1984).

${ }^{31}$ O. I. Shpotyuk, Phys. Status Solidi B 183, 365 (1994).

${ }^{32}$ C. Y. Yang, M. A. Paesler, and D. E. Sayers, Phys. Rev. B 39, 10342 (1989).

${ }^{33}$ Ke. Tanaka (unpublished).

${ }^{34}$ S. G. Bishop and N. J. Shevichik, Solid State Commun. 15, 629 (1974).

${ }^{35}$ K. O. Jensen, P. S. Salmon, I. T. Penfold, and P. G. Coleman, J. Non-Cryst. Solids 170, 57 (1994).

${ }^{36}$ K. Doi, J. Non-Cryst. Solids 51, 367 (1982).

${ }^{37}$ E. Sonder and W. A. Sibley, in Point Defects in Solids, edited by J. H. Crawford and L. M. Slifkin (Plenum, New York, 1972), Vol. 1, p. 201.

${ }^{38}$ R. Zallen, The Physics of Amorphous Solids (Wiley, New York, 1983).

${ }^{39}$ J. Wong and C. A. Angell, Glass: Structure by Spectroscopy (Marcel Dekker, New York, 1976), Chap. 9.

${ }^{40}$ K. Tanaka, Jpn. J. Appl. Phys., Part 1 25, 779 (1986).

${ }^{41}$ G. Pfeiffer and M. A. Paesler, J. Non-Cryst. Solids 114, 130 (1989).

${ }^{42}$ For the case of electron-beam-induced deformation [K. Tanaka, Appl. Phys. Lett. 70, 261 (1997)], the darkening appears earlier.

${ }^{43}$ Ke. Tanaka, J. Appl. Phys. 65, 2042 (1989).

${ }^{44}$ K. Tanaka, Oyo Buturi 66, 252 (1997).

${ }^{45} \mathrm{~S}$. Tsuchihashi and Y. Kawamoto, J. Non-Cryst. Solids 5, 286 (1971).

${ }^{46}$ Handbook of Chemistry and Physics, 74th ed., edited by D. R. Lide (CRC Press, Boca Raton, 1993).

${ }^{47}$ B. T. Kolomiets, Phys. Status Solidi 7, 359 (1964).

${ }^{48}$ Y. Kawamoto and S. Tsuchihashi, J. Am. Ceram. Soc. 54, 131 (1971).

${ }^{49}$ M. A. Stevens Kalceff, M. R. Phillips, and A. R. Moon, J. Appl. Phys. 80, 4308 (1996).

${ }^{50}$ J. A. Ruller and E. J. Friebele, J. Non-Cryst. Solids 136, 163 (1991). 